\title{
Estrategias para promover la participación de familias en la educación de niños en escuelas chilenas ${ }^{1}$
}

Alicia Razeto ${ }^{2}$

\section{Resumen}

En el tema de la relación familia-escuela se ha estudiado más sus beneficios que las estrategias para fomentarla. Por ello este artículo tiene como propósito explorar la visión que tienen directores sobre la participación parental en la educación y describir las estrategias implementadas en sus escuelas, mediante la aplicación de entrevistas a directores de veintinueve escuelas públicas básicas de la Región Metropolitana, Chile. Los resultados muestran altas expectativas hacia las familias en la definición de participación, al mismo tiempo que una evaluación crítica del estado en la que ella se encuentra en sus escuelas. También se describen las estrategias implementadas, entre las que están: actividades festivas, de esparcimiento y de celebración de las familias; instancias formales de encuentro entre familias, profesores y directores; actividades de formación integral de padres; visitas domiciliarias de los trabajadores sociales a las familias; y el uso de tecnologías de información y comunicación como forma de acercamiento a las familias. Estas estrategias son discutidas a la luz del modelo de participación parental de Joyce Epstein (2011). Se concluye que las estrategias que las escuelas implementan para fomentar la relación con las familias son limitadas, tradicionales y no reconocen la pluralidad de estructuras familiares, por lo que no están de acuerdo a los cambios que ha experimentado la sociedad chilena. A diferencia de lo propuesto por el modelo de Epstein, en las escuelas estudiadas no se refirieron a prácticas relativas al ejercicio del voluntariado de padres y profesores, a estrategias de colaboración con la comunidad, ni tampoco a experiencias que se orientasen explícitamente a apoyar el aprendizaje en el hogar.

\section{Palabras clave}

Relación familia-escuela - Participación parental en la educación - Estrategias de participación parental.

1- Este artículo es un producto académico del estudio Fondecyt de Iniciación № 11140679, Conicyt Gobierno de Chile.

2- Pontificia Universidad Católica de Chile, Santiago, Chile. Contacto: arazeto@uc.cl

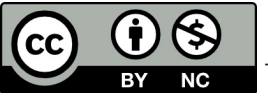




\section{Strategies to promote family participation in child education in Chilean schools}

\section{Abstract}

Regarding family-school relationship, benefits have been studied more than the strategies to promote it. For this reason, this paper intends to explore the vision that principals have of parental participation in education and describe the strategies implemented in their schools, by conducting interviews with the principals of twenty-nine public elementary schools in the Metropolitan Area, Chile. The results show high expectations towards the families in the definition of participation, at the same time as a critical evaluation of the condition in which the family is in their schools. I also describe the strategies implemented, including: festivities, recreation and celebration of the families; formal places of meeting with families, teachers and principals; activities of integral formation of parents; home visits of the social workers to the families; and the use of technologies of information and communication as a way to get closer to the families. These strategies are discussed in the light of the model of parental participation by Joyce Epstein (2011). Conclusion is that the strategies the schools implement to promote the relation with the families are limited, traditional and they do not recognize the plurality of family structures, as the strategies do not follow the changes in Chilean society. Instead of what is proposed for the model of Epstein, in studied schools, there are no references to practices relative to voluntary work of parents and teachers, to strategies of collaboration with the community.

\section{Keywords}

Family-school relationship - Parental participation in education - Strategies for family participation.

\section{Introducción}

Las familias asumen un papel significativo en el proceso de aprendizaje y socialización de los niños y adolescentes. Así lo han demostrado numerosos estudios internacionales realizados a partir de la segunda mitad del siglo XX, los cuales confirman la influencia del compromiso e involucramiento de los padres, madres o responsables de los niños en sus resultados educativos (HOOVER-DEMPSEY et al., 2006; EPSTEIN; VAN VOORHIS, 2010; HENDERSON; MAPP, 2002; HILL; CRAFT, 2003; COLEMAN, 1966; RASBASH et al., 2010).

La participación de los padres en la educación está asociada con una mejor asistencia a la escuela, puntajes más altos en matemáticas y lectura, más altas tasas de graduación en educación secundaria y menor grado de repitencia (LAROCQUE; KLEIMAN; DARLING, 
2011). También está asociado con mejores resultados en áreas no académicas, como la satisfacción de los padres y alumnos con la escuela, menores problemas de disciplina y programas escolares más efectivos. A su vez, la participación parental beneficia a la escuela como organización, pues mejora sus índices de resultados y su capacidad de gestión (SHELDON; BEE, 2015).

En Chile, el impacto de la familia en los resultados educativos se ha investigado en los resultados de las pruebas del Sistema de Medición de la Calidad de la Enseñanza SIMCE. Sus estadísticas oficiales confirman que el involucramiento de los padres o responsables de los niños en el proceso educativo se relaciona con mejores resultados de aprendizaje, existiendo hasta 23 puntos de diferencia entre establecimientos de alto y bajo involucramiento de los padres (segundo, cuarto y sexto básico, SIMCE, 2013).

A pesar de lo elocuente que ha sido la evidencia internacional acumulada por más de 40 años sobre el innegable papel predictor que tiene el involucramiento de la familia en los resultados escolares de los niños, los recursos y responsabilidades para promover significativamente este compromiso han sido pocos, débiles e inconsistentes (WEISS et al., 2009). Considerando este déficit, es fundamental desarrollar estudios enfocados a identificar y comprender las prácticas, estrategias o programas que las escuelas aplican para relacionarse con las familias, describiéndolas e interrogándolas para dotarlas de mayor efectividad e innovación. Esto orientado a impulsar el compromiso e involucramiento de las familias en la educación de los niños y adolescentes y mejorar los resultados educativos de los estudiantes.

Con esta intención, el presente artículo contiene los resultados de un estudio realizado en veintinueve escuelas públicas chilenas el año 2015, el cual tuvo como preguntas de investigación: ¿Cómo conciben la participación de las familias en la educación los directores de las escuelas? ¿Cuáles son las estrategias que aplican las escuelas para fomentar la participación parental? Considerando la naturaleza de las preguntas planteadas, es preciso señalar que el presente artículo desarrolla un abordaje generalista y exploratorio, siendo su principal aporte el describir las prácticas que se están aplicando actualmente en las escuelas chilenas para fortalecer la relación entre las escuelas y las familias. La relevancia del estudio radica en ser el primero en Chile que identifica, sistematiza y describe las estrategias más recurrentes que los directores reconocen utilizar en sus escuelas para promover la participación de los padres en la educación de sus hijos, asunto que no está completamente normado ni regulado, ni menos estandarizado entre las escuelas públicas.

\section{Referentes conceptuales}

Las transformaciones que experimenta la estructura de las familias afectan sus dinámicas y procesos, aunque mantienen intacto su valor en la educación y desarrollo de los niños: “[...] la familia constituye la institución más importante para la educación infantil en todo el mundo, y varias décadas de investigación han permitido concluir que una familia sólida fomenta el desarrollo positivo de los niños" (LIPPMAN; WILCOX, 2014, p. 3). Esta relevancia ya era 
destacada por Morandé (1999) quien plantea que, ante la funcionalización de la educación escolarizada, la familia aumenta su responsabilidad en la formación integral de las personas.

Entre la escuela y familia debiese desarrollarse una relación colaborativa, una relación de sociedad o alianza (ALCALAY et al., 2003; VILLARROEL, 2008; SIMON; ECHEITA, 2012; EPSTEIN, 2011; SIMON; GINE; ECHEITA, 2016) entre educadores, padres y otros actores de la comunidad, en la que comparten la responsabilidad por el aprendizaje y el desarrollo, siguiendo un modelo de "[...] superposición de las esferas de influencia entre la escuela, familia y comunidad para trabajar en conjunto con el propósito de guiar y apoyar el aprendizaje y desarrollo de los estudiantes.” (EPSTEIN, 2011, p. 43). Sin embargo, esta relación de alianza y colaboración enfrenta barreras, como las halladas por La Rocque, Kleiman y Darling (2011), quienes identifican las siguientes:

i) Barreras emocionales, producidas por la desconfianza entre los profesores y las familias.

ii) Barreras de lenguaje, debido a que puede parecerle a las familias científico o abstracto y hacer de la relación entre profesor y padres una experiencia impersonal, lo que puede intimidar a los padres.

iii) Barreras físicas, referidas a los problemas de traslados entre trabajo, hogar y escuela, y a dificultades en la organización del cuidado de niños.

iv) Diferencias culturales, pues las culturas difieren en la forma en la que expresan su preocupación por el niño. En consecuencia, la participación parental variará de acuerdo a la cultura étnica de origen de la familia.

En el ámbito de las diferencias culturales y sociales, también es relevante mencionar la emergencia de nuevas y diversas formas de familias. De acuerdo a Sanz y otros autores (2013) el modelo hegemónico de familia se ha visto modificado por los cambios sociales, tales como el aumento del divorcio, la incorporación de la mujer al trabajo, la disminución de la natalidad, entre otros. Así es como emergen distintas formas de hacer familia, como por ejemplo la familia monoparental, la familia recompuesta, la familia homoparental. Esta pluralidad debe ser reconocida en las discusiones sobre la alianza familia-escuela, produciendo estrategias de participación familiar que sean sensibles a esta realidad y capaces de integrarlas de modo tal de ser inclusivas y más efectivas.

Conceptualmente, el asunto de la alianza y la colaboración entre familia y escuela refiere al concepto de participación de las familias en la educación, existiendo diversas formas de definirla. La Unesco (2004, p. 28) la define como la “[...] la posibilidad de incidir, de decidir, de opinar, de aportar, de disentir y de actuar en diversos campos de la educación, acordados previamente y de común acuerdo entre docentes, padres y otros agentes educativos [...]”. Por su parte, el Harvard Family Research Project (2014) define la participación de las familias en la educación bajo tres principios claves:

- Como una forma en la cual las familias refuerzan el aprendizaje en múltiples contextos en los que los niños aprenden: en el hogar, en el jardín infantil, en la escuela, en programas de verano, en instituciones religiosas y en la comunidad. 
- Como una responsabilidad compartida en la cual la escuela y otras agencias de la comunidad se comprometen a llegar a involucrar a las familias de modo significativo y en el cual las familias se comprometen a apoyar activamente el aprendizaje y desarrollo de los niños.

- Como un continuo a lo largo de la vida del niño e implica el compromiso duradero y roles parentales cambiantes cuando los niños maduran hacia la juventud.

En esta discusión, es preciso distinguir entre participación parental en la educación y en la escuela y para esto ayuda el aporte de Overstreet y otros autores (2005). Según estos autores existen tres tipos de participación parental: la participación en la escuela, la participación cognitiva-intelectual y la participación personal. La primera se refiere a las actividades que se realizan en la escuela o actividades relacionadas con la escuela que suceden en el hogar (como la ayuda en las tareas). La segunda se refiere a la exposición intelectual de actividades estimulantes con las que los adultos enganchan a sus niños, como leer juntos. Mientras que la tercera, es acerca del conocimiento que se tiene sobre lo que ocurre con el niño en la escuela y qué es lo que está trabajando en ella. Según el estudio de estos investigadores con población afroamericana, se demostró que "[...] la participación en la escuela es el factor más importante en la motivación y desempeño académico de los niños" (OVERSTREET et al., 2005 p. 101).

LaRocque, Kleiman y Darling (2011) definen la participación parental en la educación (parental involvement) como la inversión o esfuerzo que realizan los padres o cuidadores en la educación de sus niños. Según estos autores, en la práctica este involucramiento puede ser demostrado a través de la participación de los padres en una serie de actividades tales como:

- Voluntariado en la escuela.

- Ayudar a los niños en las tareas.

- Asistir a actividades en la escuela.

- Visitar la sala de clases de los niños.

- Compartir experiencia o expertise con la clase a través de charlas.

- Asumir cargos de liderazgo en la escuela y participar en el proceso de toma de decisiones.

Es importante tener en cuenta que ciertas prácticas de involucramiento pueden ser más efectivas que otras. Por ejemplo, que las familias tengan altas expectativas, tengan metas, monitoreen el progreso y apoyen activamente el aprendizaje en el hogar hará más probable que al niño le vaya bien en la escuela (SHLEDON; BEE, 2015). Pero si se entiende a la educación como una responsabilidad compartida a través de una alianza entre las familias y escuelas, éstas no debiesen asumir un rol pasivo en el fortalecimiento de la relación, sino que por el contrario, debiesen crear e implementar estrategias para impulsar la participación activa. Ciertamente, las diferentes culturas escolares influenciarán las representaciones y modos de intervención que tienen las escuelas y sus directores, en el desarrollo de iniciativas de relación familia-escuela.

Epstein (2011) ha desarrollado un modelo de prácticas de participación de las familias en la educación en las siguientes modalidades: 
- Parentalidad (parenting): se refiere a un tipo de práctica orientada a construir condiciones en el hogar que favorezcan el aprendizaje de los niños y su mejor comportamiento en la escuela. Es importante para ello ayudar a las familias a desarrollar conocimiento y habilidades para entender a los niños en cada edad y nivel de desarrollo. Esto puede realizarse a través de actividades tales como workshop en la escuela o en otros lugares, mensajes telefónicos computarizados, cursos o capacitación para padres, implementación de programas de apoyo familiar en áreas de salud, nutrición u otros, visitas domiciliarias, reuniones en el vecindario, entre otros.

- Comunicación (communicating): este tipo de práctica apunta a diseñar y conducir formas efectivas de comunicación bilateral entre escuelas y familias acerca de los programas escolares y el progreso de los niños. Esto se puede hacer a través actividades tales como: conferencias con todos los padres al menos una vez al año, enviar semanal o mensualmente a los hogares una carpeta con el trabajo de los estudiantes para la revisión o comentarios de los padres, el uso de un esquema regular de noticias, memos, llamados telefónico, boletines, sitio web del centro y otro tipo de comunicaciones, información a los padres sobre las políticas, programas o reformas de la escuela, información a los padres sobre la seguridad de internet.

- Voluntariado (volunteering): práctica que se refiere a reclutar y organizar ayuda y apoyo de los padres, voluntarios que pueden ayudar a los profesores, administradores o niños en clases o en otras áreas. Ejemplos de actividades de esta naturaleza son: voluntarios para la sala de clases o para la escuela en general, la disponibilidad de un centro de familia en la escuela para el trabajo voluntario, reuniones y recursos para las familias, la implementación de una encuesta anual para identificar los talentos disponibles, tiempos y localización de los voluntarios, patrullas de padres u otras actividades que le den seguridad y operación a los programas de la escuela, cadenas telefónicas para proveer información a padres, entre otros.

- Aprendizaje en el hogar (learning at home): consiste en proveer información e ideas para las familias acerca de cómo ayudar a los estudiantes en el hogar con las tareas y actividades, decisiones y planificaciones relacionadas al curriculum. Actividades que pueden implementarse bajo esta práctica son: otorgar información a las familias sobre los conocimientos y habilidades requeridas por los estudiantes en cada asignatura para cada año, información sobre la política de tareas para el hogar y cómo monitorear y discutir el trabajo escolar en el hogar, información sobre cómo apoyar a los estudiantes en mejorar sus habilidades en diversas clases y evaluaciones, establecer un esquema regular de tareas que requieran que el estudiante discuta e interactúe con sus familias respecto a lo que está aprendiendo en clases, calendario con actividades para padres y estudiantes para realizar en la casa o en la comunidad, actividades familiares de lectura, ciencia y matemáticas en la escuela, actividades de aprendizaje en vacaciones, entre otras.

- Participación en la toma de decisiones (decision making): se refiere a un tipo de práctica orientada a incluir a los padres en las decisiones de la escuela, favoreciendo el desarrollo de padres líderes y representativos. Las actividades que pueden contemplarse son: activar organizaciones de padres, consejos o comités (por ejemplo, comité curriculum, 
seguridad o personal), grupos de defensa independientes para negociar y trabajar para la reforma de la escuela y su mejoramiento, consejos o comités de nivel local para el involucramiento de la familia y la comunidad, información de elecciones en la escuela, redes para unir a todas las familias con los padres representantes.

- Colaboración con la comunidad (collaborating with the community): consiste en identificar e integrar recursos y servicios desde la comunidad para fortalecer los programas de la escuela, las prácticas de las familias, y el aprendizaje de los estudiantes y su desarrollo. Las actividades posibles de realizar son: informar a estudiantes y familias de los programas y servicios de salud, culturales, recreacionales y de apoyo social que hay disponibles en la comunidad; informar de actividades comunitarias que incrementen el aprendizaje de habilidades y talentos, incluyendo los programas de verano para estudiantes, otorgar servicios a la comunidad por los estudiantes y familias, a través del reciclaje, arte, música, teatro y otras actividades; entre otros.

Para que estas formas de participación se hagan efectivas, se requiere la presencia de programas en la escuela destinados a tal fin. De acuerdo a Egido (2015) los rasgos comunes que caracterizan las experiencias de éxito de los programas de colaboración familia-escuela son:

- Consideran a padres y profesores como iguales y valoran la contribución de las familias al proceso educativo, en un clima de diálogo y confianza.

- Escuela adopta un papel proactivo y trabaja para conseguir la participación de todos los padres y también de grupos u organizaciones del entorno.

- Buscan la colaboración de padres de alumnos de todas las edades y no solo de los más pequeños, buscan fórmulas de cooperación adaptadas a las distintas etapas educativas.

- Motivan y forman a toda la comunidad educativa para entender la necesidad, utilidad y sentido de la colaboración.

- Plantean el tema de la colaboración como una cuestión de calidad más que de cantidad, evitando actividades inconexas, se realiza una planificación estratégica de las formas de colaboración y adaptables al cambio.

\section{Metodología de la investigación}

La naturaleza de las preguntas del estudio condujo a asumir un enfoque metodológico de carácter cualitativo y un tipo de estudio de nivel exploratorio-descriptivo, lo cual permitió profundizar en la descripción de las prácticas utilizadas por las escuelas para incrementar la participación de los padres en la educación.

La muestra consistió en veintinueve escuelas básicas municipales o públicas de cuatro comunas de la Región Metropolitana, previendo que cada comuna representara territorialmente una zona geográfica de la Región Metropolitana. Estas fueron: Santiago de la zona centro, La Pintana de la zona sur, Cerrillos de la zona poniente y Peñalolén de la zona oriente.

Una caracterización de las escuelas participantes del estudio se aprecia en el Tabla 1. El Índice de vulnerabilidad social (IVE) es un indicador de vulnerabilidad 
social y riesgo de los estudiantes de cada establecimiento. Por su parte, el SIMCE es el Sistema nacional de medición de la calidad de la educación, que evalúa el desempeño de los estudiantes. Lo hace censalmente todos los años, en distintos sectores y niveles de aprendizaje.

Tabla 1- Caracterización de las escuelas participantes del estudio

\begin{tabular}{|c|c|c|c|c|c|}
\hline Comunas & $\begin{array}{c}\text { Cantidad } \\
\text { de escuelas } \\
\text { participantes }\end{array}$ & $\begin{array}{l}\text { Cantidad de } \\
\text { alumnos promedio } \\
\text { (matrícula anual) }\end{array}$ & $\begin{array}{l}\text { Cantidad de } \\
\text { profesores } \\
\text { promedio }\end{array}$ & $\begin{array}{c}\text { Indice de } \\
\text { vulnerabilidad } \\
\text { escolar }(\%)^{*}\end{array}$ & $\begin{array}{l}\text { Simce } 4^{0} \text { básico } \\
\text { Comprensión } \\
\text { Lectura }(2015)^{* *}\end{array}$ \\
\hline Santiago & 8 & 637 & 44 & 62,4 & 252 \\
\hline La Pintana & 7 & 317 & 32 & 90,5 & 233 \\
\hline Cerrillos & 6 & 338 & 28 & 84,1 & 240 \\
\hline Peñalolén & 8 & 381 & 34 & 84,2 & 243 \\
\hline
\end{tabular}

Fuente: Elaboración propia en base a datos de MINEDUC y JUNAEB (CHILE, 2017a, 2017b).

* El promedio nacional del IVE Basica 2017 fue de 80,5\%.

** El promedio nacional del SIMCE $4{ }^{\circ}$ Básico del año 2015 en Comprensión de Lectura fue de 265 puntos.

Posteriormente, se hizo un catastro del total de escuelas básicas municipales existentes en cada comuna, para que la muestra alcanzara un nivel de cobertura de un 70 a un $80 \%$. Un criterio fundamental de selección que los encargados municipales de educación y fundamentalmente los directores expresaran su disposición voluntaria para participar del estudio. Con vista a estos criterios, la muestra quedó conformada por la siguiente cantidad de escuelas:

Tabla 2- Informantes, escuelas y comunas participantes del estudio

\begin{tabular}{|c|c|c|}
\hline Comuna & Cantidad de escuelas & Cantidad de informantes \\
\hline Santiago & 7 & $\begin{array}{l}6 \text { directores } \\
1 \text { subdirector }\end{array}$ \\
\hline Peñalolén & 8 & $\begin{array}{l}7 \text { directores } \\
1 \text { jefe de UTP }\end{array}$ \\
\hline La Pintana & 7 & $\begin{array}{l}5 \text { directores } \\
1 \text { jefe de UTP } \\
1 \text { inspector }\end{array}$ \\
\hline Cerrillos & 6 & 6 directores \\
\hline Total & 28 escuelas & 28 entrevistados \\
\hline
\end{tabular}

Fuente: Elaboración propia.

La estrategia de recolección de información fue la entrevista semi-estructurada al director del establecimiento educativo. Se seleccionó al director como informante principal por ser quien asume el liderazgo administrativo y pedagógico, y quien decide y autoriza 
la puesta en marcha de las diversas iniciativas o actividades en la escuela. Esta entrevista contempló ocho preguntas abiertas, que fueron las siguientes:

1) ¿Cómo son las familias de los niños de esta escuela?

2) ¿Qué significado o sentido tiene para usted la idea de participación de las familias en la educación de los niños?

3) ¿Cómo se imagina la participación ideal de las familias en la educación de sus hijos?

4) ¿Cuáles son las prácticas o iniciativas que tiene la escuela para involucrar la participación de los padres en la educación de sus hijos en el hogar?

5) ¿Se ha gestado alguna iniciativa propia de la escuela, más allá de lo solicitado por el Ministerio?

6) ¿Tienen conocimiento de los resultados de estas iniciativas? ¿Cómo son evaluadas por usted?

7) ¿Cuáles son las principales dificultades u obstáculos que han enfrentado las iniciativas de participación de padres en la escuela? Distinguir obstáculos provenientes de la escuela y de las familias.

8) ¿Hay algún profesional de la escuela que asuma las funciones de relación familiaescuela? ¿Trabajador social?

La entrevista tuvo una duración promedio de 45 minutos y en todos los casos fue aplicada al interior de las dependencias de las escuelas, usualmente en la oficina del director.

Antes de cada entrevista, se presentó un formulario de consentimiento informado, al cual todos los directores accedieron firmar. Tras la obtención del permiso respectivo, las entrevistas fueron grabadas digitalmente y transcritas por el equipo de ayudantes de la investigación.

El análisis de la información recolectada se hizo a través de análisis de contenido y para la codificación se utilizó el software NVIVO 10. Las categorías de análisis fueron principalmente deductivas, creadas a partir de los objetivos del estudio y dirigidas por la pauta de preguntas de la entrevista aplicada. Estas son: definición de participación de familias en la educación y estrategias de participación aplicadas en las escuelas.

\section{Resultados del estudio}

En esta sección se presentan dos ámbitos principales de resultados: la visión que tienen los directores sobre la participación parental en la educación y las estrategias que aplican en sus escuelas para promoverla.

Participación de las familias en la educación de los niños: ¿cómo la definen los directores?

Los directores comprenden la participación de las familias en la educación de las siguientes formas, las que están ordenadas partiendo por aquellas más presentes en sus discursos: 
- Que las familias asistan y colaboren con actividades organizadas y realizadas en la escuela: por ejemplo, que asistan a la reunión de padres y apoderados ${ }^{3}$; a las citaciones por parte de profesores $u$ otros profesionales; que participen del centro de padres $y$ apoderados; que colaboren en diversas actividades que se organizan durante el año.

- Que las familias respondan con responsabilidad a las exigencias de la escuela: asegurando la asistencia regular y puntual del niño a clases; asegurando que el niño presente los materiales requeridos; asegurando una retirada puntual de los alumnos; revisando la libreta de comunicaciones, cuadernos y tareas.

- Que las familias, estableciendo límites y normas, cumplan sus responsabilidades parentales de cuidado y satisfacción de sus necesidades: procurando que los niños se alimenten correctamente; que duerman la cantidad de horas necesarias para funcionar adecuadamente al día siguiente; que demuestren higiene personal.

- Que las familias proporcionen en el hogar las condiciones mínimas para facilitar el estudio de los niños estableciendo horarios de estudio, un lugar adecuado para realizar las tareas y estudiar, entre otros.

En estas definiciones se puede constatar que los directores tienen altas expectativas en el rol de los padres, asumiendo una postura exigente hacia ellos desde una relación jerárquica, que no expresa una intención de apoyo o colaboración en el cumplimiento de las expectativas, lo cual sería relevante considerando que las escuelas participantes atienden mayoritariamente a estudiantes de familias de condiciones socioeconómicas vulnerables. Tampoco se observa un discurso sensible a la diversidad de formas de hacer familia, sino que más bien los directores mantienen un discurso centrado en la noción de familia a la usanza tradicional.

En las definiciones más recurrentes, está presente la premisa que las familias deben ser las que se acercan a la escuela y no ésta a las familias, a pesar de las dificultades que mencionan se presentan en esta relación: "Lo ideal es que se acercaran a la escuela que cómo van sus hijos, como están, en qué nos pueden aportar o nosotros, que se acerquen por último decir que no sé hacer esto pero que mis niños puedan hacerlo [...]" (DM224).

Los directores son categóricos al momento de evaluar la forma en la que las familias participan en la educación, con una visión principalmente crítica, como lo refleja esta directora:

[...] las familias en general, en general creo yo que son bien ausentes de sus hijos, de las realidades educativas de sus pupilos, por lo tanto dificulta bastante el tema académico pedagógico porque en realidad solo vienen a dejar a cargo al niño y sólo es nuestra responsabilidad educarlo, nos traspasan la educación de sus hijos en términos generales a la escuela "y de mal a peor" yo noto que los niños de primero básico por ejemplo, súper abandonados, no tienen hábitos de casa [...] (DM17).

3- En Chile, el término apoderados se utiliza para referirse a los responsables de los niños ante la escuela. Puede corresponder al padre, madre u otro adulto a cargo del niño.

4- Las citaciones que aparecen de aquí en adelante corresponden a fragmentos de las entrevistas realizadas para esta investigación con directores de distintas escuelas. La letra D identifica el cargo laboral de director y la M o H su sexo, siendo M mujer o sexo femenino y $\mathrm{H}$ hombre o sexo masculino. Por su parte, el número identifica a la individualidad del director entrevistado, siendo ocupado en lugar del nombre con el fin de resguardar su anonimato. 
Predomina en los directores la visión que los padres conciben a la escuela principalmente como un lugar de cuidado, protección y seguridad para sus hijos, pero no como un espacio para la educación en el que tienen algún grado de responsabilidad, lo cual se relacionaría con el bajo involucramiento en la escuela: “[...] la gran mayoría nos ve como eso, nos ve como el lugar donde sus niños están bien cuidados, donde están bien protegidos, pero más allá digamos, no logramos que el apoderado sea parte nuestra en esto [...]" (DH25).

\section{Estrategias implementadas por las escuelas para promover la participación de las familias en la educación de sus niños}

En primer lugar, es importante señalar que no se detectaron programas establecidos implementándose en esta temática dentro de las escuelas participantes de este estudio, sino que más bien iniciativas y actividades que ya sea por tradición o por necesidad son realizadas a lo largo del año escolar.

Asimismo, se encontraron prácticas mayoritariamente históricas, a pesar de que algunas escuelas se estaban abriendo a utilizar estrategias nuevas mediante el uso de tecnologías y redes. Principalmente se trata de prácticas realizadas por tradición más que por convicción o por el resultado de evaluaciones formales.

El Cuadro 1 muestra las principales prácticas destacadas por los directores en lo que refiere a la relación entre escuelas y familias. Todas ellas, a excepción de las visitas domiciliarias y las estrategias de comunicación a través de tecnologías, se llevan a cabo en las dependencias físicas de las escuelas.

Cuadro 1- Estrategias aplicadas en las escuelas para promover la participación parental en la educación de los niños

\begin{tabular}{|c|c|c|}
\hline 동 & \multirow{4}{*}{ Actividades festivas, de esparcimiento y celebraciones } & Deportivas \\
\hline ర్ల్ర & & Artísticas \\
\hline ․ㅡㄹ & & Recreativas \\
\hline$\stackrel{\widetilde{్}}{\varrho}$ & & De homenaje \\
\hline \multirow{9}{*}{ 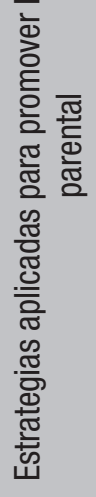 } & \multirow{4}{*}{ Instancias formales de encuentro entre familias, profesores y directivos } & Reuniones \\
\hline & & Entrevistas/citaciones \\
\hline & & Centro de padres \\
\hline & & Consejo escolar \\
\hline & Formación integral de padres & \\
\hline & Visitas domiciliarias a las familias & \\
\hline & \multirow{3}{*}{ Uso de tecnología como medio de información } & Mensajería de texto \\
\hline & & Sitios web \\
\hline & & Redes sociales \\
\hline
\end{tabular}

Fuente: Elaboración propia. 


\section{Actividades festivas, de esparcimiento y celebraciones}

Estas actividades son organizadas por la escuela y realizadas en su infraestructura. Las familias de los estudiantes son invitadas abierta y masivamente a presenciar las demostraciones de los estudiantes y participar de ellas. El 75\% de los directores entrevistados informaron que en sus escuelas se ejecutaban algunas de las actividades mencionadas en las siguientes categorias:

1) Actividades artísticas: de alta relevancia para la escuela en las cuales los estudiantes muestran a sus familiares una performance artística preparada y ensayada con antelación durante el semestre. Entre estas se destaca el acto de fiestas patrias, encuentros folclóricos o muestra folclórica anual, aniversario de la escuela, celebración del 21 de mayo, fiesta de la primavera, feria de la multiculturalidad, kermes y gala artística.

2) Actividades recreativas: tales como paseos, bingos solidarios, concursos de cueca, fiestas de disfraces, fiesta de fin de año, almuerzo familiar, caravanas, talleres de flores de bach y campañas ecológicas o verdes.

4) Actividades de reconocimiento u homenaje: como la celebración de los días de la mujer, la madre, del niño, del padre, del alumno, del abuelo y la celebración del día de la graduación.

3) Actividades de naturaleza deportiva: campeonatos de atletismo y fútbol para padres y sesiones de zumba.

Es relevante señalar que este tipo de actividades fueron las más mencionadas por los directores entrevistados al momento de preguntarles por las prácticas en la relación entre familia y escuela. La razón de ello obedecería a que son las que logran la mayor asistencia y participación de los padres. En otras palabras, serían las más valoradas, en razón del entusiasmo que provoca la posibilidad de vivir un momento de fiesta, de compartir con otros, esparcimiento y alegría. Así lo refleja esta directora: “[...] donde veo que se acercan muchos apoderados, son actividades masivas, pero son actividades de orden recreativas. Por último, se vinculan con sus hijos y con la escuela. Eso es donde más apoderados llegan” (DM22).

Esta situación no está libre de críticas por parte de algunos directores, que perciben una contradicción entre la asistencia a este tipo de actividades y las de carácter más académico:

[...] te digo lamentablemente o felizmente no sé, por ejemplo, eh... para venir a pasarlo bien están todos, la kermes, 18 de septiembre, que se yo, pero cuando quieres conversar de ellos de los aprendizajes de los niños [...] (DM16).

[...] los padres por ejemplo son muy participativos si hacemos un almuerzo familiar. Nosotros hacemos un almuerzo familiar, que es un día sábado a la hora de almuerzo aquí. Y viene la familia completa, comen y lo pasan bien, disfrutan. Eso tiene convocatoria, eso tiene mucha convocatoria, pero no así tanto las reuniones.

E: Ya, ¿y a qué lo asocia Ud. eso?

D: A que quieren pasarlo bien ...seguramente están muy aproblemados en sus vidas, igual hay gente aquí que tienen historias bien aproblemadas, y no te dan mucha diversión por aquí. No les dan muchas oportunidades de pasarlo bien por aquí cerca. La escuela ofrece una oportunidad (DH10) 


\section{Instancias formales de encuentro entre familias, profesores y directivos}

Hay cuatro instancias oficiales de encuentro entre estos miembros de la comunidad educativa que fueron destacadas con frecuencia por los directores entrevistados: reunión de padres, entrevistas o citaciones de profesores a padres, la participación de estos en el centro de padres y el consejo escolar.

\section{Reunión de padres y apoderados}

Estas reuniones son mencionadas por el 78\% de los directores entrevistados e indicadas como las principales actividades formales que la escuela realiza con las familias de los estudiantes. Principalmente constituyen una instancia de encuentro periódica entre padres o responsable de los niños y escuelas conducentes a entregar información y orientaciones a las familias acerca del progreso o dificultades generales reportadas por el grupo curso. Son organizadas por el equipo de la escuela, generalmente por los profesores, y son realizadas en la infraestructura escolar en los horarios que la escuela escoja, por lo general, vespertinos. Suelen realizarla una vez al mes o una vez cada dos meses.

Uno de los aspectos críticos develados por los directores es la asistencia de los padres o responsables a estas reuniones. En algunas escuelas los directores afirmaron tener una baja asistencia siendo su principal explicación la incompatibilidad de días y horarios entre los profesores y los padres o responsables que están trabajando. Adicionalmente, se detecta que hay factores etarios y climáticos que influyen en la asistencia, siendo en los niveles más bajos y en verano y primavera cando hay un mejor indicador. Sin embargo, también hay discursos más críticos que asumen que la baja asistencia se debe a la forma en la cual las reuniones de organizan y realizan, orientadas a solicitar dinero para financiar actividades y a informar.

Otros directores reportan una buena tasa de asistencia que obedece a un proceso de mejoramiento activo de la calidad de las reuniones que la escuela ha realizado:

Dejamos de hacer una reunión como para cobrar dinero, cambiamos el enfoque de eso, reuniones de... no se cobra dinero y nosotros creamos una estructura para la reunión. Tiene tres partes la reunión, donde el pro... todos los cursos siguen la misma parte, es una reunión pareja para todos y al profesor se le entrega, la dupla psicosocial que trabaja con nosotros, que es la psicóloga con la trabajadora social, ellos son los que arman la reunión" (DM26).

Una de las cosas que nos proponemos tratar de mejorar es el clima, el carácter de las reuniones de apoderados que no se conviertan en una lata, en una queja porque ese es un motivo más que hace que el apoderado no sienta mayor interés, o sea, para ir a escuchar de que se quejaron de su hijo, que le agregaron más problemas, que está todo malo. Entonces hay que buscar un mecanismo, de cómo hacemos reuniones atractivas, que realmente el apoderado se sienta a gusto en escuela, que no sea un desagrado" (DH1). 


\section{Entrevista 0 citaciones de padres con profesores}

$\mathrm{Al}$ igual que las reuniones, se trata de una instancia de encuentro entre el profesor y el padre o responsable, que se organiza desde la escuela -mayoritariamente es solicitada por el profesor y no por el padre- y se realiza en sus dependencias físicas. A diferencia de las reuniones, los directores destacan que las entrevistas o citaciones tienen la ventaja de proveer un acercamiento individual entre el profesor y el padre, que produce un encuentro de mayor intimidad y confianza para conocerse y conversar con mayor profundidad. Los directores manifestaron realizarse dos veces por semestre. El 32\% de los directores entrevistados informaron acerca de esta estrategia.

[...] están destinadas algunas horas no lectivas de los profesores para entrevistar a los papás de todos los alumnos, entonces ahí vamos pesquisando las necesidades de cada uno, yo creo que esa entrevista que es más personal con los papás y con los niños nos da muchos más antecedentes, porque en realidad en las reuniones generales, las asambleas generales que hacemos con los padres los papás no se abren mucho a su problemática, entonces cuando se da un espacio más de intimidad ahí logramos saber bien que es lo que pasa, los docentes hacen esas reuniones que tienen como obligatorias y también los profesionales que están trabajando en la escuela, la asistente social, los psicólogos, la mediadora, cuando es pertinente hacerla. (DM17).

Si bien los directores no profundizan en la forma en la cual se llevan a cabo ni en los logros que producen, mencionan dificultades para contactar a los padres, coordinar los tiempos entre el profesor y el padre, y lograr finalmente la asistencia de estos a la entrevista. "[...] ellos acá son atendidos cuando nosotros los mandamos a buscar, son muy pocos los apoderados que vienen voluntariamente a saber cómo es el proceso educativo de sus hijos" (DM23).

\section{Centro de padres}

El 57\% de los directores mencionan al centro de padres y apoderados como una organización presente en la escuela, como una instancia de apoyo y colaboración hacia las estrategias educativas de la escuela. Sin embargo, también dan cuenta de las situaciones de baja participación de las familias en este espacio, de problemáticas en la constitución o rotación de directivas y de conflictos internos.

\section{Consejo escolar}

El Consejo Escolar es un órgano principalmente informativo y consultivo, que tiene por propósito "propender a la activa participación de todos los actores de la comunidad escolar con el objeto de mejorar la calidad de la educación y los logros de aprendizaje en los establecimientos educacionales" (CHILE, 2005, p. 1). A pesar de que la norma establece que es propositivo, no se especifican los aspectos en los que puede proponer. A su vez, este organismo podría llegar a ser resolutivo, siempre y cuando el sostenedor así 
lo decida. Hubo un 25\% de directores que mencionaron el Consejo Escolar como canal de participación de los padres en una instancia formal de toma de decisiones en la escuela, resolutiva en algunas materias, en la que participan los padres, en su calidad de miembros del centro de padres y apoderados.

\section{Formación integral de padres y apoderados}

La escuela para padres es la principal iniciativa desplegada, consistente en encuentros colectivos o talleres con padres y responsables de los niños que se realizan con distinta periodicidad durante el año para trabajar con ellos diferentes temas relacionados con la crianza de los niños y su educación integral. Esta actividad fue mencionada con bastante frecuencia por los directores participantes del estudio (82\%). Las temáticas tratadas en estas actividades están orientadas a prevenir o remediar diversas problemáticas de los estudiantes, tales como el alcoholismo y consumo de drogas, educación sexual, alimentación sana, buen trato, ciberbullying y habilidades parentales. Por lo general, los directores señalan que son realizadas por los profesionales que componen la dupla psicosocial (psicólogo y trabajador social) o en algunos casos, por profesionales externos de diversos programas sociales. La periodicidad varía de escuela en escuela, siendo las más frecuentes realizadas una vez al mes y las menos una vez al semestre. Respecto a los horarios, algunas escuelas las realizan en el horario que antecedente a la reunión de apoderados y otros manifiestan realizarla durante estas reuniones.

Si bien son actividades valoradas por los directores y catalogadas como necesarias para el buen funcionamiento de la relación entre familia y escuela, también es cierto que adolecen de algunas dificultades en su funcionamiento siendo las principales la asistencia de los responsables de los niños y la falta de evaluación de su impacto.

\section{Visitas domiciliarias}

Fue reconocida como práctica implementada en el establecimiento por el 53\% de los directores entrevistados. Esta estrategia es valorada por los directores como un canal de comunicación entre las familias y las escuelas, e implica un traslado de un o un conjunto de profesionales al hogar del estudiante. En la mayoría de las escuelas es realizada por la dupla psicosocial, particularmente la trabajadora social. Aunque en el caso de una de las comunas la labor era realizada por un programa externo a la escuela de carácter municipal.

Mayoritariamente los directores informan ser utilizada frente a situaciones de inasistencia a la escuela, o vulneración de derechos de estudiantes. Cuando el mecanismo del llamado telefónico no ha dado resultados, entonces se realiza la visita al hogar para conocer las razones de la inasistencia e incentivar el reintegro a clases del estudiante.

Respecto a sus resultados hay opiniones distintas, en algunos casos se reportan buenos resultados y en otras variables, siendo las principales dificultades que en ocasiones los padres no permiten la entrada de la profesional al hogar y en otras hay rechazo a la visita porque se sienten fiscalizados. 


\section{Tecnologías de información como estrategia de comunicación a las familias}

Esta es una práctica instalada recientemente en algunas escuelas (28\%) participantes del estudio. Como estrategia de comunicación con las familias, mencionan la disposición de un sitio web institucional con información relevante, y en algunos casos el uso de Facebook institucional, y de un correo institucional. Ocasionalmente se reporta el caso de profesores que se comunican via whatsapp con sus padres y apoderados.

En una de las comunas estudiadas las escuelas destacan un programa de mensajería de texto, apoyado por una empresa externa, que permite enviar notas, recordatorio de fechas de pruebas y reuniones, y otras observaciones a los padres o responsables de los niños.

\section{Discusión y conclusiones}

Los resultados del estudio muestran que los directores suelen poseer una visión ideal sobre la participación parental altamente exigente, funcional a la operación cotidiana de la escuela más que en el desarrollo integral de los niños, centrada en el deber ser de los padres y poco empática en la expresión de apoyo frente a las dificultades que presentan las familias de condición social vulnerable para involucrarse en la educación de sus hijos. Tampoco demuestran tener una visión sensible a las diversas estructuras familiares, propias de la sociedad moderna, sino que aplican un criterio común de participación familiar sin considerar la pluralidad existente ni las dificultades que experimentan las familias de acuerdo a su propia realidad.

No se encontraron discursos afines con la idea de incidencia en la toma de decisiones escolares (UNESCO, 2004) ni tampoco una definición que concibiera la participación como una responsabilidad compartida entre la escuela y la familia (HARVARD PROJECT FAMILY, 2014). A su vez, se percata que las definiciones brindadas por los directores están enfocadas en el tipo de participación en la escuela y no en los otros tipos de participación descritos por Overstreet y otros autores (2005).

Los directores valoran la participación parental en la educación, pero pareciera ser que han experimentado el desencanto al presenciar las dificultades para que ella sea una realidad positiva en la escuela. En este sentido, las barreras identificadas por LaRocque, Kleiman y Darling (2011) podrían verse reflejadas en las realidades escolares estudiadas, especialmente las emocionales y físicas. Paradójicamente, la mayoría de los directores parecieran estar estancados en este estado de desencanto, reproduciendo una relación distante con las familias en lugar de asumir estrategias más decididas y planificadas para promover la participación parental.

La visión predominantemente crítica que tienen los directores de la participación de las familias en la educación podría conducir a deteriorar el clima abierto y cordial que se requiere para incentivar la participación de los padres. El estudio realizado por Overstreet y otros autores (2005) en población afroamericana desventajada socialmente, determinó que uno de los predictores más potentes de participación parental en la escuela es la receptividad de la escuela, es decir, que los padres sientan que esta los escucha y organiza actividades significativas. La desconfianza instalada en la relación entre las 
familias y la escuela se contradice con la principal conclusión del estudio de Peña (2000) que recomienda que para aumentar la participación de los padres en la educación de sus niños es hacerlos sentir bienvenidos, confiar en ellos y reconocer las ventajas que ofrecería trabajar colaborativamente con ellos.

Con relación a la descripción de las estrategias aplicadas en las escuelas para incrementar la participación parental, se encontró cierta gama de diversidad en el grupo de escuelas estudiadas, pero al interior de cada una eran dos las prácticas reconocidas: las actividades festivas, de esparcimiento y celebraciones y las instancias formales de encuentro entre familias, profesores y directores. Las actividades festivas eran las más frecuentemente nombradas y a las que se les atribuye mayor éxito por la masividad de la asistencia de las familias de los niños. Sin embargo, desde el punto de vista de su impacto en los resultados educativos, la participación en este tipo de iniciativas es poco decidora. De hecho, el modelo de Epstein (2011) no las incorpora en ninguno de los niveles deseables de participación parental. Distinto es el caso de las instancias formales de encuentro entre las familias, profesores y directores, que sí son afınes con el nivel de participación de comunicación propuesto por Epstein (2011). Sin embargo, varios directores revelaron que adolecen de dificultades operativas relativas a la baja asistencia y participación de los padres, como es el caso de las reuniones, citaciones y participación en centro de padres. Respecto al Consejo Escolar, en teoría afín con el nivel de toma de decisiones del modelo de Epstein (2011), es una instancia que sólo en algunos temas resuelve, es decir, su posibilidad de toma de decisiones es restringida.

Por otra parte, de acuerdo al modelo de Epstein (2011) la formación integral de padres sería una actividad relevante desde el punto de vista de su potencial aporte a la parentalidad, a la comunicación y al aprendizaje en el hogar. No obstante, lo directores informaron adolecer de problemas en la asistencia de los padres, siendo realizadas esporádicamente a lo largo del año escolar.

Por su parte, las visitas domiciliarias, si bien fueron mencionadas por más del $50 \%$ de los directores entrevistados, se utilizan como un mecanismo reactivo ante la inasistencia de los niños a la escuela y no como una estrategia de acercamiento entre ambos actores que permita comprometer a las familias en la educación de sus niños. Cabe señalar que ante las dificultades reportadas como el trabajo de los padres, las distancias, y la vulnerabilidad social de las familias, la visita domiciliaria se presenta como una estrategia socioeducativa de alto potencial.

En definitiva, se encontraron en su mayoría estrategias más bien tradicionales y con poca innovación o introducción de modificaciones producto de evaluaciones. No se constató que las escuelas adoptaran un papel proactivo en la incorporación de nuevas estrategias de colaboración, ni tampoco un programa de relación familia-escuela instalado, que reflejase un proceso de planificación estratégica de las formas de colaboración y que fuesen adaptables al cambio, tal como lo sugiere Egido (2015).

Podría decirse que las estrategias que las escuelas implementan para fomentar la relación con las familias son limitadas y coincidentemente con lo indicado por la UNESCO (2004) son tradicionales y que no están de acuerdo a los cambios que ha experimentado la sociedad chilena. No se encontraron prácticas relativas al ejercicio del voluntariado 
de padres y profesores en las escuelas, a estrategias de colaboración con la comunidad, ni tampoco experiencias que se orientasen explícitamente a apoyar el aprendizaje en el hogar, tal como lo sugieren Epsetin (2011) y LaRocque, Kleiman y Darling (2011).

Las limitaciones de este estudio consisten en que sólo se haya entrevistado a los directores de las escuelas. Pese a ser un informante fundamental, también hubiese sido deseable entrevistar a profesores y a padres o responsables de los niños, para conocer su visión respecto a la temática trabajada en este artículo. Otra limitación tiene que ver con el tamaño de la muestra de directores y la imposibilidad de generalizar los resultados del estudio.

Finalmente, para futuras investigaciones se podría considerar la pertinencia de complementar y profundizar el tema de estudio desde la perspectiva de padres y profesores. También sería relevante realizar estudios de efectividad de las estrategias implementadas o de evaluación de su impacto.

\section{Referencias}

ALCALAY, Lidia et al. Familia y escuela. ¿Una alianza posible? Una mirada desde la perspectiva de los estudios. Psykhe, Santiago de Chile, v. 12, n. 2, p. 101-110, 2003.

CHILE. Junta Nacional de Auxilio Escolar y Becas (Junaeb). Prioridades 2017 con IVE SINAE básica, media y comunal. Santiago de Chile: Junaeb, 2017a. Disponible en: <https://www.junaeb.cl/ive> . Acceso en: 11 nov. 2017.

CHILE. Ministerio de Educación (Mineduc). Decreto 24 reglamenta consejos escolares. Santiago de Chile: Mineduc, 2005. Disponible en: <https://www.leychile.cl/Navegar?idNorma=236237>. Acceso en: 09 sept. 2016.

CHILE. Ministerio de Educación (Mineduc). Más información, mejor educación. Santiago de Chile: Mineduc, 2017b. Disponible en: <http://www.mime.mineduc.cl/mvc/mime/portada>. Acceso en: 02 nov. 2017.

COLEMAN, James. Equality of educational opportunity study. Washington, DC: Government, 1966.

EGID0, Inmaculada. Las relaciones entre familia y escuela. Revista del Consejo Escolar del Estado, Madrid, v. 4, n. 7. p. 11-17, 2015.

EPSTEIN, Joyce. School, family and community partnerships. Boulder: Westview Press, 2011.

EPSTEIN, Joyce; VAN V0ORHIS, Frances. More Than minutes: teachers' roles in designing homework. Educational Psychologist, v. 36, n. 3, p. 181-193, 2010. D0I: 10.1207/S15326985EP3603_4.

HARVARD FAMILY RESEARCH PROJECT. Redefining family engagement for student success. Charlestown: [s. n.], 2014. Disponible en: <http://www.hfrp.org/family-involvement/publications-resources/redefiningfamily-engagement-for-student-success>. Acceso en: 29 dic. 2016. 
HENDERSON, Anne; MAPP, Karen. A new wave of evidence: the impact of school, family, and community connections on student achievement. Austin: TX, Southwest Educational Development Laboratory, 2002.

HILL, Nancy; CRAFT, Stracie. Parent-school involvement and school performance: mediated pathways among socioeconomically comparable African American and Euro-american families. Journal of Educational Psychology, Washington, DC, v. 95, n. 1, p.74-83, 2003.

HOOVER-DEMPSEY, Kathleen et al. Why do parents become involved? Research findings and implications. The elementary School Journal, Chicago, v. 106, n. 2, p. 105-130, 2006.

LAROCQUE, Michelle; KLEIMAN, Ira; DARLING, Sharon. Parental involvement: the missing link in school achievement. Preventing School Failure, Philadelphia, v. 55, n.3, p.115-122, 2011.

LIPPMAN, Laura; WILCOX, Bradford. World family map 2014: mapping family change and child well-being outcomes. Washington, DC: [s. n.], 2014. Disponible en: <http://www.childtrends.org/?publications=worldfamily-map-2014-mapping-family-change-and-child-well-being-outcomes>. Acceso en: 11 sept. 2016.

MORANDÉ, Pedro. Familia y sociedad: reflexiones sociológicas. Santiago de Chile: Universitaria, 1999. (Orientaciones en ciencia, tecnología y cultura).

OVERSTREET, Stacy et al. Predicting parental involvement in children's schooling within an economically disadvantaged african american sample. Psychology in the Schools, Indiana, v. 42, n. 1, p. 101-111, 2005.

PEÑA, Delores. Parent involvement: influencing factors and implications. The Journal of Educational Research, Philadelphia, v. 94, n. 1, p. 42-54, 2000.

RASBASH, Jon et al. Children's educational progress: partitioning family, school and area effects. Journal of the Royal Statistical Society, London, v. 173, n. 3, p. 657-682, 2010. Series A.

SANZ, Jesús et al. Diversidad familiar: apuntes desde la antropología social. Revista de Treball Social, Barcelona, n. 198, p. 30-40, 2013.

SHELDON, Steven; BEE, Sol. The family engagement partnership student outcome evaluation. Baltimore: Johns Hopkins University, 2015. Disponible en: <http://flamboyanfoundation.org/wp/wp-content/ uploads/2015/09/JHU-STUDY_FINAL-REPORT.pdf>. Acceso en: 20 dic. 2015.

SIMCE. Sistema de Medición de la Calidad de la Enseñanza. Resultados nacionales Simce. Santiago de Chile: Simce, 2013. Disponible en <http://www.agenciaeducacion.cl/biblioteca-digital/resultados-simce/>. Acceso en: 02 en. 2016.

SIMÓN, Cecilia; ECHEITA, Gerardo. La alianza entre las familias y la escuela en la educación de alum nado más vulnerable. Revista Padres y Maestros, Madrid, n. 344, p. 31-34, 2012.

SIMÓN, Cecilia; GINÉ, Climent; ECHEITA, Gerardo. Escuela, familia y comunidad: construyendo alianzas para promover la ilnclusión. Revista Latinoamericana de Educación Inclusiva, Santiago de Chile, v. 10, n. 1, p. 25-42, 2016. 
UNESCO. Participación de las familias en la educación infantil latinoamericana. [S. I.]: Unesco, 2004. Disponible en: <http://unesdoc.unesco.org/images/0013/001390/139030s.pdf>. Acceso en: 20 mzo. 2017.

VILLARROEL, Gladys. La alianza familia - escuela como una condición necesaria para lograr mejores aprendizajes. Perspectivas Educacionales, Valparaiso, n. 7, p. 49-63, 2008.

WEISS, Heather et al. Reframing family involvement in education: supporting families to support educational equity. Equity Matters, New York, n. 5, dic. 2009. Disponible en: <https://files.eric.ed.gov/fulltext/ED523994. pdf>. Acceso en: 7 en. 2017.

Recibido en: 25.05.2017

Revisiones: 24.08.2017

Aprobado en: 25.10.2017

Alicia Razeto es doctora en Ciencias de la Educación de la Escuela de Trabajo Social, Facultad de Ciencias Sociales, Pontificia Universidad Católica de Chile y magíster en Gestión y políticas públicas de la Universidad de Chile. Investigadora responsable del Fondecyt 11140679. Académica de la Escuela de Trabajo Social, Facultad de Ciencias Sociales de la Pontificia Universidad Católica de Chile. 